\title{
Statine verbessern offenbar SSRI-Therapie
}

\author{
Statinen wird nachgesagt, dass sie die antidepressive Wirkung von SSRI verstärken können. \\ Eine große Registerstudie aus Dänemark liefert neue Hinweise für diese Vermutung.
}

_ Für eine populationsbasierte Kohortenstudie wurden die Daten aller dänischen Patienten analysiert, die von 19972012 neu auf einen selektiven SerotoninWiederaufnahmehemmer (SSRI) eingestellt wurden - und die irgendwann in dieser Zeit gleichzeitig ein Statin zur Cholesterinsenkung eingenommen hatten. Ermittelt wurde, ob die Patienten psychiatrische Krankenhäuser aufsuchten, suizidales Verhalten zeigten oder verstarben.

Von den 872.216 Patienten, die auf einen SSRI eingestellt wurden, erhielten 113.108 (13\%) gleichzeitig ein Statin. Die Kombinationsbehandlung führte im Vergleich mit alleiniger SSRI-Gabe zu einer signifikanten Risikoerniedrigung sowohl bezüglich psychiatrischer Klinikkontakte beliebiger Ursache (Hazard Ratio [HR] 0,75, 95\%-Konfidenzintervall
[KI] 0,69-0,82) als auch speziell wegen einer Depression (HR 0,64, 95\%-KI 0,55-0,75). Dabei fanden sich weder beim allgemeinen Mortalitätsrisiko (HR 1,04; 95\%-KI 0,96-1,12) noch beim Auftreten suizidalen Verhaltens (HR 0,85; 95\%-KI 0,61-1,18) signifikante Unterschiede. Die Autoren schließen daraus, dass Statine die antidepressive Effektivität von SSRI steigern könnten.

- Köhler O, Gasse C, Petersen L et al. The Effect of Concomitant Treatment With SSRIs and Statins: A Population-Based Study. Am J Psychiatry 2016;173:807-15

\section{KOMMENTAR}

In bisherigen klinischen Studien zeigten sich bereits antidepressive Augmentationseffekte von Simvastatin und Lovastatin. Die Stichproben waren jedoch relativ klein, und hinsichtlich einer Remission wurde keine Wirksamkeit nachgewiesen.
Nun spricht die verbesserte Wirkung einer SSRI-Statin-Kombinationsbehandlung auf die globalen Parameter in der vorliegenden Studie grundsätzlich für eine klinische Relevanz. Zudem ergab sich für die Kombinationsbehandlung kein erhöhtes allgemeines Mortalitätsrisiko. Spezifisch für Lovastatin war die Mortalität allerdings erhöht. Eine erhöhte Suizidalität fand sich hingegen weder allgemein noch unter spezifischen Kombinationsbehandlungen. Zur Beurteilung der Wirksamkeit hinsichtlich spezifischer Substanzen war lediglich für die Kombination Citalopram mit Simvastatin eine ausreichende statistische Power gegeben.

Man darf natürlich nicht vergessen, dass die Satine nicht primär die antidepressive Therapie augmentieren sollten, sondern dass es sich um Koinzidenzen handelt. Trotzdem erscheinen weitere Studien gerechtfertigt.

Prof. Dr. med. C. Lange-Asschenfeldt

\section{Nach der TAVI wandern Cholesterinkristalle in die Zehen}
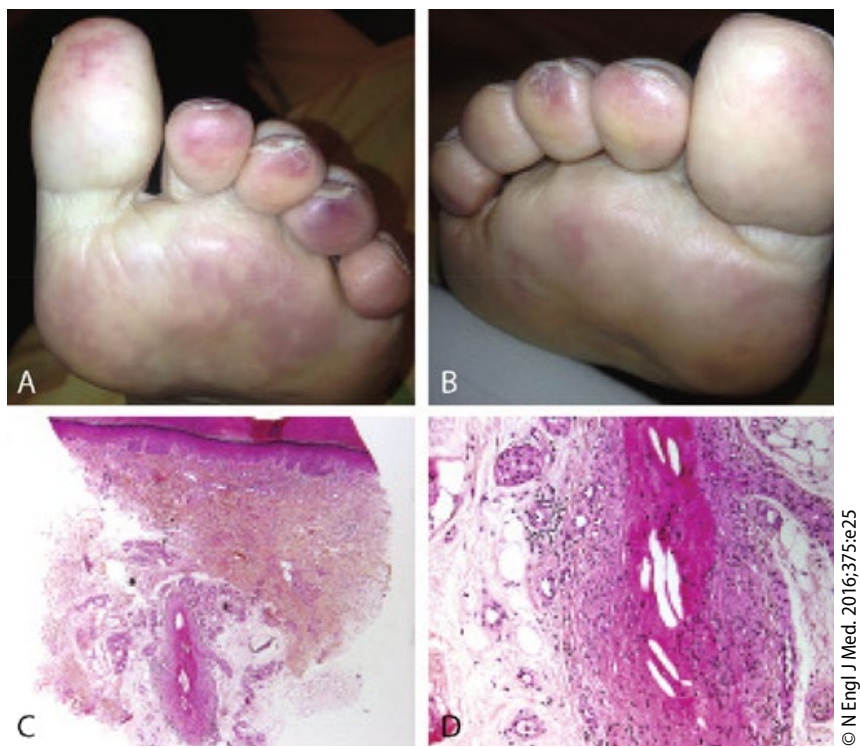

A, B: Fleckige, bläulich-livide Verfärbungen an den Zehen. C, D: Cholesterinkristalle in den mittelgroßen Arterien.
Eine 88-jährige Frau mit Zustand nach Vierfach-Bypass stellte sich wegen fleckiger, bläulich-livider Verfärbungen an den Zehen vor. Vier Wochen zuvor hatte sie sich einer Transkatheter-Aortenklappenimplantation (TAVI) unterzogen. Die Hautverfärbungen erinnerten an eine Livedo reticularis (Abb. A, B). Im Labor wies sie einen erhöhten Serum-Kreatininwert von 2,8 $\mathrm{mg} / \mathrm{dl}$ auf, der von initial 1,4 $\mathrm{mg} / \mathrm{dl}$ angestiegen war. Es bestand eine leichte Leukozytose mit einer Eosinophilie von 13\%. In der Hautbiopsie zeigten sich obstruktive Cholesterinkristalle in den mittelgroßen Arterien (Abb. C, D). Dieser Befund bestätigte die Diagnose eines Cholesterinemboliesyndroms.

Dieses Syndrom wird hervorgerufen durch arterielle Verschleppung von Cholesterinkristallen in die Peripherie. Es können zahlreiche Organsysteme einbezogen werden, darunter vor allem periphere Gefäße, Nieren und Gehirn. Es handelt sich um eine bekannte Komplikation von Angiografien, gefäßchirurgischen Eingriffen, thrombolytischen Therapiemaßnahmen und, in jüngster Zeit zunehmend, der TAVI. Die Hautveränderungen bildeten sich im vorliegenden Fall binnen drei Wochen zurück. Die Nierenfunktion hingegen normalisierte sich nicht mehr vollständig.

Prof. Dr. med. H. S. FüeßI

- Devy L, Brunet-Possenti F (devy.lu87@yahoo.fr): Cholesterol embolization after transcatheter aortic-valve replacement. N Engl J Med. 2016;375:e25 